\title{
RESSECÕES HEPÁTICAS POR VIDEOLAPAROSCOPIA
}

\author{
Current status of laparoscopic liver resections \\ Paulo HERMAN, Fabricio Ferreira COELHO, Renato Micelli LUPINACCI, Marcos Vinicius PERINI, \\ Marcel Autran C. MACHADO, Luiz A. Carneiro D'ALBUQUERQUE, Ivan CECCONELLO
}

ABCDDV/678

Herman P, Coleho FF, Lupinacci RM, Perini MV, Machado MAC, D’Albuquerque LAC, Cecconello I. Ressecões hepáticas por videolaparoscopia. ABCD Arq Bras Cir Dig 2009;22(4):226-32

RESUMO - Introdução - As ressecções hepáticas representam umas das últimas fronteiras vencidas pela cirurgia videolaparoscópica. Apesar da complexidade do procedimento, da demanda de grande incorporação de tecnologia e necessidade de experiência em cirurgia hepática e laparoscópica, a indicação do método tem crescido de forma expressiva nos últimos anos. Objetivo - Realizar análise crítica do método, baseada nos trabalhos existentes na literatura, ressaltando o estado atual de suas indicações, exequibilidade, segurança, resultados e aspectos técnicos primordiais. Método - Foram identificados e analisados os trabalhos pertinentes nas bases de dados LILACS e PUBMED até dezembro de 2009, utilizando-se os descritores "liver resection", "laparoscopic" e "liver surgery". Não foram encontrados trabalhos prospectivos e randomizados sobre o tema, sendo os dados disponíveis provenientes de série de casos, estudos caso-controle e alguns estudos multicêntricos e metanálises. Conclusão - A hepatectomia por videolaparoscopia é hoje operação segura e factível, mesmo para as ressecções hepáticas maiores, com baixo índice de morbimortalidade. O método pode ser utilizado para lesões malignas sem prejuízo dos princípios oncológicos e com vantagens nos pacientes com cirrose ou disfunção hepática. A melhor indicação recai sobre as lesões benignas, em especial o adenoma hepatocelular. Em mãos experientes e casos selecionados, como as lesões benignas localizadas nos segmentos anterolaterais hepáticos, principalmente no segmento lateral esquerdo, a ressecção videolaparoscópica pode ser considerada hoje como tratamento padrão.

DESCRITORES - Hepatectomia. Laparoscopia. Cirurgia hepática. Revisão

\section{INTRODUÇÃO}

Os avanços técnicos da cirurgia videolaparoscópica (VL) transformaram a terapêutica cirúrgica de grande número de doenças. Com a constante evolução e advento de novas tecnologias a VL tem sido empregada com bons resultados na ressecção de diversos órgãos intra-abdominais e retroperitoneais, tais como estômago, cólon, rins, pâncreas, adrenal e baço ${ }^{12}$. No tratamento de algumas doenças, como colecistolitíase e doença do refluxo gastroesofágico, transformaram-se na terapêutica de escolha com vantagens inquestionáveis sobre a abordagem convencional ${ }^{41}$.

Na cirurgia hepática, o emprego da via VL está bem estabelecido em alguns procedimentos como biópsias hepáticas, destelhamento de cistos hepáticos simples e drenagem de abscessos ${ }^{17}$. No entanto, as ressecções hepáticas representam umas das últimas fronteiras vencidas pela cirurgia $\mathrm{VL}^{7,12,13}$.

Os fatores para essa dificuldade passam pelo risco

Trabalho realizado no Serviço de Cirurgia do Fígado e Hipertensão Portal, Departamento de Gastroenterologia, Universidade de São Paulo, São Paulo, SP, Brasil

Endereço para correspondência: Paulo Herman, e-mail: pherman@uol.com.br teórico de embolia gasosa, potencial de sangramento intraoperatório incontrolável, incertezas sobre a obtenção de margens cirúrgicas adequadas e risco de disseminação tumoral nos casos de doenças malignas, além da necessidade de grande incorporação de tecnologia, como a necessária para secção do parênquima hepático (bisturi harmônico, grampeadores vasculares laparoscópicos, etc), transdutores laparoscópicos para ultrassonografia intra-operatória e necessidade de afastadores e instrumentais específicos para a mobilização hepática. Adicionalmente a curva de aprendizado do método é longa, exigindo cirurgiões com experiência em cirurgia hepática e formação em cirurgia VL avançada $a^{7,13,23}$.

A primeira hepatectomia VL foi publicada por Gagner at al. ${ }^{16}$ em 1992 para o tratamento de hiperplasia nodular focal hepática e, em 1995, Ferzl et al. ${ }^{14}$ reportaram a ressecção de um adenoma hepático de $9 \mathrm{~cm}$ no segmento IV. A primeira hepatectomia regrada foi publicada por Azagra et $\mathrm{al}^{1}$. em 1996, que realizaram uma segmentectomia lateral esquerda em paciente com adenoma hepático. Ulteriormente a essas experiências iniciais, trabalhos subsequentes restringiam-se a relatos e séries de casos de poucos centros especializados. Só mais recentemente, em especial nos últimos cinco anos, é que os trabalhos sobre o tema cresceram de forma expressiva $a^{1,7,24,38}$. 
Diferentes séries retrospectivas e estudos de casocontrole demonstraram que a ressecção laparoscópica é factível, segura e com benefícios adicionais sobre a abordagem convencional. Dentre as vantagens das ressecções hepáticas VL estão menor dor pós-operatória, menor tempo de internação, menor tempo de retorno ao trabalho e menor índice de hérnias incisionais ${ }^{30,38}$. Vários autores observaram ainda, menor índice de sangramento intra-operatório nos pacientes submetidos à ressecção hepática VL quando comparado à via laparotômica ${ }^{30}$. Além disto, as complicações intra e pós-operatórias relatadas para as ressecções VL são semelhantes as da cirurgia convencional, com taxas semelhantes de morbidade e mortalidade ${ }^{12,13,30,38}$.

Impulsionada pelas vantagens supracitadas e pelos bons resultados obtidos quando comparada às hepatectomias convencionais, a via de acesso VL atualmente tem sido utilizada para a ressecção de lesões hepáticas benignas e malignas $^{18,24,29}$. O aumento da experiência com o método e o desenvolvimento de novas tecnologias tem demonstrado que os problemas técnicos das grandes ressecções hepáticas podem ser contornados. Por essa razão, os limites têm sido ampliados, com a realização de ressecções hepáticas maiores (mais de três segmentos) com sucesso ${ }^{1,35}$.

O objetivo da presente revisão é realizar análise crítica do método, baseada nos trabalhos existentes na literatura, ressaltando o estado atual das indicações, exequibilidade, segurança, resultados e aspectos técnicos das ressecções hepáticas VL empregadas no tratamento das doenças benignas e malignas do fígado.

\section{INDICAÇÕES}

Apesar do aumento das indicações, o emprego da VL nas ressecções hepáticas ainda é restrito, sendo na maioria dos centros, a via de acesso de escolha em 5 a $30 \%$ de todas as hepatectomias. Em alguns serviços ultra-especializados percentual de 50 a $80 \%$ tem sido relatado ${ }^{40}$.

\section{Doenças benignas}

A VL é utilizada nas ressecções de lesões hepáticas benignas e malignas ${ }^{18,29}$. Os tumores benignos são aqueles que têm sua melhor indicação e foram as responsáveis pelo desenvolvimento inicial do método na cirurgia hepática. Dentre as lesões benignas pode-se destacar o destelhamento VL de cistos hepáticos simples, que tem sido largamente utilizado e já se tornou procedimento consagrado. Os cistos hidáticos também têm relatos de tratamento por VL, utilizando aspiração e injeção de substâncias escolicidas ${ }^{10}$. Os cistoadenomas biliares ${ }^{17}$, pelo seu risco de transformação maligna, têm indicação de ressecção e a via VL pode ser utilizada em casos selecionados.

A indicação mais aceita na literatura para ressecção hepática VL é a de tumores benignos, sendo os melhores candidatos os portadores de tumores periféricos e com possibilidade de ressecção limitada do parênquima ${ }^{38}$.

Dentre as lesões benignas, os hemangiomas e as hiperplasias nodulares focais pelo seu comportamento indolente devem ser apenas acompanhados e a excisão cirúrgica só é indicada nos tumores sintomáticos, compressivos ou em crescimento ${ }^{19,20,38}$. Por outro lado, conduta mais agressiva deve ser empregada no tratamento dos adenomas hepatocelulares pelo seu risco comprovado de rotura e malignização ${ }^{22}$, sendo essa a melhor indicação dentre os tumores benignos.

As recomendações clássicas para utilização do método são nas lesões anterolaterais do fígado (segmentos 2, 3, 4b, 5,6 ), também chamados por alguns autores de "segmentos laparoscópicos" ${ }^{" 30,40}$. As ressecções hepáticas menores nesses segmentos têm sido consideradas seguras e de fácil execução. Alguns autores propõem a utilização rotineira nas segmentectomias laterais esquerdas (segmentos 2 e 3 ) a VL pelos seus bons resultados, facilidade técnica e baixos índices de complicações ${ }^{6}$. Já as ressecções maiores ainda não têm seu uso disseminado, no entanto, o aumento da experiência e o advento de novas tecnologias fizeram com que as dificuldades técnicas das grandes ressecções hepáticas pudessem ser suplantadas e possibilitou a realização de ressecções hepáticas maiores, como hepatectomias direita e esquerda ou mesmo trissegmentectomias ${ }^{1,35}$. Alguns serviços têm realizado hepatectomia direita de rotina com bons resultados ${ }^{33}$. Mais recentemente, tem-se aplicado o método também nas ressecções para a retirada de enxertos parciais de doadores vivos de fígado ${ }^{25,37}$.

A possibilidade de oferecer tratamento cirúrgico adequado com menor morbidade abre grande espaço para o uso do método com vantagens evidentes principalmente nos pacientes com doenças benignas que são, em geral, mulheres em faixas etárias mais jovens. Nesse sentido, existe número crescente de séries na literatura atestando a exequibilidade e benefícios da ressecção VL para tumores hepáticos benignos com resultados bastante favoráveis ${ }^{12,25,30,38}$.

Deve-se sempre salientar, no entanto, que o emprego da videolaparoscopia não deve ampliar as indicações de ressecção dos tumores benignos, devendo ser utilizadas as mesmas da cirurgia convencional ${ }^{40}$.

\section{Transplante hepático}

Atualmente, um dos assuntos de maior interesse e mais controversos na cirurgia hepática tem sido as hepatectomias para doadores vivos. A mortalidade para este procedimento varia de $0,5 \%$ a $1,0 \%$ dependendo do centro e do tipo de ressecção hepática. A experiência inicial começou com ressecções do setor lateral esquerdo para transplantes pediátricos ${ }^{37}$. Com a evolução contínua da cirurgia hepática minimamente invasiva a técnica começou a ser aplicada para hepatectomias direitas em doadores adultos ${ }^{2}$, sendo utilizada a cirurgia vídeo-assistida por vários centros nos Estados Unidos e no Japão. Até agora nenhuma mortalidade foi relatada em hepatectomias laparoscópicas para doadores vivos seja para transplante pediátrico ou adulto, e as complicações aconteceram em proporções semelhantes ao procedimento convencional ${ }^{5}$. No entanto, mais dados são necessários para confirmar os resultados da cirurgia minimamente invasiva no transplante de fígado. 


\section{Doenças malignas}

As indicações de hepatectomia laparoscópica não são diferentes daquelas da cirurgia aberta. No caso das doenças malignas, as metástases hepáticas de câncer colorretal (MHCCR) e o carcinoma hepatocelular (CHC) têm sido as principais indicações ${ }^{1,4,29}$. Poucos casos de ressecções por colangiocarcinomas periféricos e metástases de tumores não colorretais foram relatados ${ }^{4}$. Controvérsias sobre a laparoscopia em pacientes com câncer surgiram de relatos iniciais com níveis inaceitáveis de implantes peritoneais e nos portos de entrada dos trocárteres de videolaparoscopia, em especial no câncer da vesícula biliar e nos primeiros relatos de colectomias $\mathrm{VL}^{15}$. Entretanto, a estrita obediência aos princípios oncológicos como não manipulação direta do tumor, conversão imediata se câncer localmente avançado, e proteção para extração do espécime cirúrgico reduziu esse problema e hoje nas principais séries não existe diferença na frequência de disseminação tumoral entre as vias VLe convencional ${ }^{1,4,24}$.

Até o presente momento, a via laparoscópica é contraindicada na maioria dos centros, em casos de câncer da vesícula biliar e colangiocarcinomas hilares por causa do risco relatado de disseminação tumoral peritoneal e a necessidade de ressecções extensas, respectivamente ${ }^{15,24}$.

É importante ressaltar que o uso da ultrassonografia laparoscópica é de grande utilidade para a definição de margens cirúrgicas adequadas ${ }^{36}$.

Nos primeiros trabalhos publicados sobre ressecções hepáticas por laparoscopia a porcentagem de lesões benignas era largamente superior a de tumores malignos ${ }^{7,13}$. Entretanto a proporção de tumores malignos vem aumentando progressivamente nos últimos anos e hoje já perfazem metade das indicações ${ }^{1,4,24,31}$.

Assim como para as lesões benignas, o principal critério utilizado na tentativa de selecionar os melhores candidatos para as ressecções hepáticas VL é localização tumoral, sendo os locais preferenciais os segmentos anterolaterais do fígado. O tamanho da lesão apesar de importante na programação cirúrgica e as recomendações apontarem para lesões menores que $5 \mathrm{~cm}^{1,4}$, não é contraindicação formal exceto quando tumores muito volumosos em situação anatômica desfavorável, pela dificuldade de mobilização do tumor e pelo risco de ruptura da lesão ou margem limítrofe $^{5,24,40}$.

\section{Tumores primários}

A ressecção hepática é o tratamento de escolha para o $\mathrm{CHC}$ em pacientes com cirrose compensada em muitos centros. Nos pacientes cirróticos, ressecções hepáticas, mesmo pequenas, apresentam um risco importante de descompensação da função hepática no pós-operatório e complicações relacionadas, incluindo ascite, icterícia e encefalopatia. Benefícios específicos do acesso VL são a preservação da parede abdominal e sua circulação colateral venosa, diminuindo assim o regime de hipertensão portal no pós-operatório, menor necessidade de reposição volêmica e melhor reabsorção da ascite pela preservação peritoneal ${ }^{7}$. Alguns grupos têm demonstrado benefício das ressecções laparoscópicas para pequenos $\mathrm{CHC}$ em cirróticos, com diminuição da morbidade, em especial na ocorrência de ascite pós-operatória ${ }^{3,8,11,26}$. Dois estudos caso-controle compararam os resultados de ressecções hepáticas VL e abertas em pacientes cirróticos. Foi observada diminuição da morbidade, em especial menor formação de ascite pós-operatória, mas também tendência de menor perda sanguínea, período de hospitalização menor nos pacientes operados por laparoscopia, enquanto o tempo operatório foi maior ${ }^{3,8}$.

A ressecção laparoscópica de pequenos $\mathrm{CHC}$ superficiais em fígado cirrótico é factível e segura em centros especializados. Apesar de outros tratamentos além da ressecção de pequenos nódulos isolados de $\mathrm{CHC}$ tenham levado a bons resultados, as ressecções laparoscópicas fornecem uma análise completa da histologia do tumor, e a possibilidade de identificação de fatores de pior prognóstico, tais como, tumores pouco diferenciados, presença de invasão micro ou macrovascular e nódulos satélites.

Apesar dos bons resultados, a cirurgia hepática em pacientes cirróticos deve ser considerada uma operação de risco, mesmo se realizada por VL, já que são bem estabelecidas as maiores taxas de morbidade e mortalidade relatadas em pacientes cirróticos submetidos à VL quando comparados a pacientes não cirróticos.

\section{Metástases hepáticas de câncer colorretal}

A indicação de ressecção VL das MHCCR passa pela avaliação da ressecabilidade das mesmas e das condições clínicas do paciente, que devem permitir a realização de anestesia geral e pneumoperitôneo prolongado. Pacientes que não são candidatos a ressecções abertas não devem ser candidatos a ressecções laparoscópicas.

Quanto aos pacientes apresentando múltiplas MHCCR, estes podem ser submetidos à ressecções VL se todas as lesões puderem ser ressecadas com margens livres e preferencialmente com uma única hepatectomia anatômica. A via VL deve ser evitada se ressecções múltiplas, complexas e/ou bilobares são necessárias.

Apesar dos resultados iniciais favoráveis até o momento $^{32,33}$, não existem trabalhos prospectivos que demonstrem claramente que as ressecções hepáticas VL de metástases de câncer colorretal tenham os mesmos resultados a longo prazo que as ressecções convencionais.

\section{ASPECTOS TÉCNICOS}

A hepatectomia VL é um procedimento trabalhoso e complexo, que necessita de instrumental adequado e exige cirurgiões com experiência em cirurgia hepática e formação em cirurgia VL avançada. Para sua indicação, independente do tipo de ressecção, cabe sempre o bom senso da equipe que deve pesar os riscos e benefícios oferecidos pelo método ao paciente.

Os cuidados perioperatórios devem ser rigorosos com 
adequada avaliação do paciente. A equipe anestésica deve estar afeita às particularidades da cirurgia hepática e a utilização da monitorização hemodinâmica invasiva da pressão venosa central e arterial é recomendada.

As melhores indicações para a laparoscopia nas ressecções hepáticas são as nodulectomias, ressecções em cunha ou segmentectomias de lesões localizadas nos segmentos anterolaterais do fígado. Os segmentos 1, 4a, 7 e 8 têm acesso mais restrito pelo método, assim como as ressecções de lesões próximas ao hilo ou justapostas às veias hepáticas. Cho et al. ${ }^{9}$ recentemente publicaram série de 36 pacientes com lesões localizadas nos segmentos postero-superiores (S7, S8, S4a e S1) ressecadas por acesso puramente laparoscópico. Da mesma maneira, ressecções laparoscópicas do segmento 1 foram recentemente relatadas ${ }^{5,24}$. Apesar dos relatos de sucesso, nessas situações a tentativa de ressecção deve ser realizada apenas em casos selecionados e por cirurgiões experientes. Alguns autores propõem a cirurgia vídeo-assistida (cirurgia híbrida) ou com o auxílio da mão ("hand-assisted") para tumores nessas localizações.

As ressecções hepáticas maiores, como hepatectomias direita e esquerda, podem ser realizadas com segurança, havendo relatos até de trisegmentectomias realizadas com sucesso $^{24}$. Alguns grupos já sistematizaram as grandes ressecções e as realizam com frequência ${ }^{1,33}$. No entanto, as ressecções maiores VL estão restritas a poucos centros especializados e não existem, até o momento, dados suficientes na literatura que coloquem a VL como padrão nesse tipo de situação.

Apesar da maioria dos relatos na literatura abordar a ressecção de poucos tumores com até $5 \mathrm{~cm}$, o tamanho ou a quantidade de lesões não são contra-indicações formais isoladamente à realização da videolaparoscopia ${ }^{5}$. No entanto, dependendo da localização, lesões maiores podem causar dificuldades para a adequada mobilização hepática e controle vascular do segmento a ser ressecado, além de poder apresentar um risco maior de rotura.

A técnica operatória deve obedecer às regras e cuidados tomados para as ressecções hepáticas convencionais A mobilização hepática adequada e controle vascular são pontos essenciais para o sucesso do procedimento. Por essa razão, a utilização de pinças e afastadores específicos facilitam sobremaneira o procedimento. $O$ controle vascular sempre foi uma das grandes preocupações nas hepatectomias VL, no entanto, ele pode ser feito de forma semelhante à cirurgia por via laparotômica. A dissecção hilar e isolamento dos vasos dos pedículos hepáticos direito e esquerdo podem ser realizados com segurança, bem como o isolamento das veias hepáticas. A manobra de Pringle pode ser realizada com facilidade, sendo utilizada rotineiramente por alguns autores, principalmente em ressecções maiores. Recentemente, também se reportou a segurança e factibilidade do controle intra-parenquimatoso dos pedículos glissonianos por videolaparoscopia ${ }^{27,28}$. Nas ressecções não regradas, a utilização da manobra de hemi-Pringle, por meio da colocação de pinças vasculares laparoscópicas no pedículo do lobo hepático ipsilateral é um artifício valioso na tentativa de diminuir o sangramento intra-operatório ${ }^{21}$.

O pneumoperitônio, em geral com dióxido de carbono (CO2), é outro fator que diminui o sangramento intra-operatório, principalmente venoso. A pressão intra-abdominal durante o ato operatório é mantida entre 12 e $14 \mathrm{mmHg}$, mas pode ser aumentada para coibir sangramentos com baixo risco de embolia gasosa. A pressão venosa central deve ser mantida pela equipe anestésica o mais baixa possível, idealmente abaixo de $5 \mathrm{mmHg}$, para minimizar o risco de sangramento.

Outros pontos importantes na cirurgia VL são a liberação do fígado da veia cava inferior, procedimento que quando realizado por VL propicia melhor visualização da veia do que com a cirurgia convencional; a secção do parênquima hepático, que pode ser realizada com várias formas de energia como o bisturi harmônico, ligasure, bisturi ultrassônico, eletrocautério e bisturi bipolar associados ao uso de clipes metálicos ou grampeadores vasculares laparoscópicos em vasos de maior calibre. As veias hepáticas e os pedículos vasculares devem preferencialmente ser selados com grampeadores vasculares laparoscópicos.

No caso de tumores malignos, além dos aspectos técnicos já discutidos é necessário seguir os princípios oncológicos para evitar a disseminação tumoral. Dentre as principais medidas estão a não manipulação direta do tumor, conversão imediata se tumor localmente avançado, e proteção para extração do espécime cirúrgico da cavidade.

A retirada da peça cirúrgica pode ser realizada por meio de ampliação da incisão de um dos portais ou nos espécimes maiores por meio de incisão de Pfannestiel. Após a retirada da peça é mandatória a revisão cuidadosa da hemostasia.

\section{Cirurgia com auxílio da mão ou cirurgia vídeo-asssistida}

A cirurgia com o auxílio da mão foi proposta para aumentar as indicações da cirurgia minimante invasiva hepática, bem como para aumentar a segurança do procedimento, principalmente nas grandes ressecções e nos casos submetidos à reoperações. As vantagens do método são a manutenção da sensação tátil, perdida na operação totalmente laparoscópica, a palpação e delimitação da margem de ressecção de lesões e ajuda no controle de sangramentos por compressão direta. Além disso, alguns autores utilizam o método para acesso às lesões em segmentos posteriores hepáticos ou próximos ao hilo ou grandes vasos ${ }^{5,24}$. Apesar de suas vantagens, não existe consenso sobre seu uso.

A cirurgia vídeo-assistida ou híbrida também foi proposta para aumentar as indicações da cirurgia minimamente invasiva hepática e suas vantagens cosméticas e pósoperatórias pela menor incisão na parede abdominal. Em geral, a mobilização hepática é realizada por laparoscopia e então feita pequena incisão no plano mediano ou subcostal direito para realização dos tempos subsequentes de dissecção e secção do parênquima hepático. A cirurgia híbrida ainda não tem papel definido na cirurgia hepática, podendo haver alguma vantagem em grandes procedimentos. Alguns centros utilizam a cirurgia híbrida para realização de hepatectomias em doadores vivos de fígado ${ }^{25}$. 


\section{RESULTADOS}

Para os tumores benignos hepáticos as vantagens da VL são mais evidentes, sendo este o grande grupo beneficiário do método. Para os tumores malignos ainda existem questões em aberto quanto aos seus resultados, $\mathrm{o}$ que abordaremos adiante.

Desde os primeiros relatos, têm aumentado as experiências com o método para ressecção de lesões hepáticas benignas e malignas e diferentes séries demonstraram que a ressecção laparoscópica é factível, segura e com benefícios sobre a abordagem convencional ${ }^{30,38}$. Uma metanálise de oito estudos não randomizados demonstrou menor perda sanguínea e menor tempo de internação nos pacientes submetidos à ressecção hepática laparoscópica ${ }^{25}$. Em estudo caso-controle, Troisi et al. ${ }^{38}$ compararam o uso de ressecção laparoscópica e convencional para o tratamento de lesões hepáticas benignas, observando menor tempo para realimentação, menor tempo de internação e menor índice de hérnias incisionais no grupo submetido à VL. Assim como Morino et al..$^{30}$, também em estudo caso-controle observaram menor índice de sangramento intra-operatório e menor tempo de internação nos pacientes submetidos à ressecção laparoscópica.

Com relação ao tempo operatório das ressecções videolaparoscópicas, este parece ser maior durante a curva de aprendizado, havendo diminuição progressiva com o aumento da experiência ${ }^{39}$. Alguns autores não encontraram diferença no tempo operatório quando comparado às hepatectomias convencionais. Com relação aos custos da cirurgia laparoscópica apesar dos diretos torná-la mais cara, os benefícios do método podem tornar o procedimento VL mais efetivo do ponto de vista econômico.

Os resultados oncológicos das ressecções VL do CHC laparoscópicas foram recentemente alvo de várias publicações. A margem cirúrgica foi adequada na maioria dos relatos: com uma média de mais de $1,0 \mathrm{~cm}$ em quase todas as séries publicadas e com poucos casos de margens cirúrgicas comprometidas ${ }^{3,4,5}$. Em diferentes estudos as taxas de sobrevida em três anos e de sobrevida livre de doença foram de 65 a $75 \%$ e 50 a $70 \%$, respectivamente. Estes resultados são similares, ou mesmo superiores aos relatados nas séries abertas. Nenhuma recidiva em portos da laparoscopia foi observada

A ressecção é o tratamento padrão para as MHCCR e o único considerado como terapia curativa. No entanto, existem poucos trabalhos que abordam especificamente os resultados das ressecções VL nesse grupo de pacientes ${ }^{29,33}$. Apesar de ressecções de MHCCR estarem presentes em outros artigos de ressecções laparoscópicas, os dados na maioria das vezes, são muitas vezes heterogêneos e incompletos. O'Rourke et al. analisaram série de 22 pacientes: a margem cirúrgica foi superior a $1,0 \mathrm{~cm}$ em $54 \%$ dos casos e a sobrevida em dois anos foi de $67 \%{ }^{33}$.

Recentemente Nguyen et al. relataram os resultados de 109 pacientes operados em cinco centros de excelência em cirurgia hepática e experiência em ressecções laparoscópi- cas. Hepatectomias maiores foram realizadas em $45 \%$ dos pacientes e complicações ocorreram em 11,9\%. Margens negativas foram obtidas em 94,5\% dos pacientes. Após seguimento médio de 20 meses ( 0,1 - 90,8 meses), 73,4 \% dos pacientes estavam vivos, e $68,8 \%$ livres de doença. A sobrevida atuarial em 1, 3, e 5 anos foi de $88 \%, 69 \%$, e $50 \% 32$.

Embora promissores, a análise destes resultados deve levar em consideração o fato de que as séries com ressecção VL envolvem pacientes altamente selecionados, com doença metastática limitada, enquanto as séries com ressecções laparotômicas de MHCCR incluem pacientes com doença mais extensa e procedimentos mais complexos. Por essa razão, um maior número de estudos é necessário para que se possam delimitar com maior precisão os resultados das ressecções VL para MHCCR.

\section{Complicações e mortalidade}

As complicações relatadas para as ressecções laparoscópicas são semelhantes às da cirurgia convencional, com taxas de morbidade entre $5 \%$ a $34 \%$ e mortalidade de 0 a $5,9 \%{ }^{12,13,30,38}$.

Apesar do risco de sangramento incontrolável ser um dos grandes óbices da cirurgia laparoscópica e a principal causa de conversão, um baixo índice de sangramento também tem sido demonstrado por vários autores, inclusive com taxas menores que a cirurgia convenciona ${ }^{25,30}$. A explicação para esse fato passa pela melhoria do instrumental utilizado na secção hepática, magnificação das imagens o que facilita a identificação das estruturas vasculares e o pneumoperitônio que coíbe o sangramento, principalmente venoso.

Outro risco da cirurgia laparocópica é o de embolia gasosa, que pode ocorrer principalmente por lesões de veias hepáticas. No entanto sua ocorrência na prática clínica é muito rara ${ }^{12,38}$. Na realidade existem poucos casos dessa complicação na literatura, havendo correlação em alguns deles com a utilização de bisturi de argônio. Por essa razão, o pneumoperitônio com $\mathrm{CO} 2$ é considerado seguro, não havendo dados que restrinjam seu uso.

A maioria dos autores relata índices de conversão que variaram de $3,3 \%$ a $15 \%^{12,13,30,38}$. As principais razões para conversão são sangramento e dificuldades técnicas. A principal causa de conversão é o sangramento intra-operatório não controlável pelo método, com frequências variáveis podendo chegar a mais de $70 \%$ dos casos. As dificuldades técnicas mais comumente relacionadas com conversão são: impossibilidade de exposição adequada do segmento a ser ressecado ou outras dificuldades anatômicas (como fígado muito grande), localização desfavorável da lesão ou risco de rotura tumoral.

Algumas séries comprovam o efeito da "curva de aprendizado" na frequência de conversões, com diminuição significante da mesma nos últimos casos quando comparados aos casos iniciais ${ }^{39}$. Quando necessária a conversão pode ser feita para a via convencional ou com auxílio da mão.

Os óbitos relacionados ás ressecções VL são raros, em revisão recente Nguyen et al. encontraram nove óbitos 
registrados em 2804 ressecções hepáticas, o que dá uma taxa de mortalidade de $0,3 \%$, sendo a principal causa de óbito a falência hepática pós-operatória em pacientes cirróticos e não havendo relatos de óbitos intra-operatórios ${ }^{31}$.

\section{CONCLUSÃO}

A ressecção videolaparoscópica hepática é um procedimento complexo que requer cirurgiões com experiência em cirurgia hepática e laparoscópica avançada, mas que tem se difundido, principalmente nos últimos anos e hoje não deve mais ser considerado um "procedimento experimental", mas sim, fazer parte do armamentário terapêutico do cirurgião de fígado.

As indicações para sua utilização devem ser as mesmas da cirurgia convencional e nesse contexto se mostrou técnica factível e segura, mesmo para as ressecções hepáticas maiores, com baixo índice de morbi-mortalidade. Em mãos experientes e em casos selecionados, com lesões benignas em segmentos anterolaterais hepáticos, principalmente no segmento lateral esquerdo, pode ser considerado como padrão-ouro de tratamento.

Os resultados oncológicos iniciais para os pacientes com lesões malignas metastáticas parecem promissores não havendo maior índice de margens cirúrgicas inadequadas ou disseminação tumoral. Nos pacientes cirróticos apesar das dificuldades técnicas, existe grande campo para a laparoscopia pela menor agressão cirúrgica e um potencial menor risco de descompensação da doença hepática no período pós-operatório.

Herman P, Coleho FF, Lupinacci RM, Perini MV, Machado MAC, D’Albuquerque LAC, Cecconello I. Current status of laparoscopic liver resections. ABCD Arq Bras Cir Dig 2009;22(4):226-32

ABSTRACT - Introduction - Hepatic resection is the last frontier to be surpassed by laparoscopic surgery. Although a highly complex procedure, the need of advanced technology and experience in both laparoscopic and hepatic surgery, the indications and number of cases done worldwide had a major growth in the last few years. Aim - Critically analyze the technique, based on published articles and acquired experience with more than 50 laparoscopic hepatic resections. Indications, feasibility, safety, and basic technical aspects are outlined. Methods - Original published studies were identified by searching the Lilacs and Medline databases (up to December 2009) using the keywords "liver resection", "laparoscopic" and "liver surgery". It was not found any prospective randomized trial, so all data came from case series, case-control studies, and meta-analysis. Conclusion - Laparoscopic liver resection is safe and feasible even for major resections, with low morbidity and mortality rates. Laparoscopic approach is considered to be oncologically similar to its open counterpart and may have some advantage in cirrhotic patients. Benign lesions, especially hepatocellular adenoma, remains the best indication. In experienced centers the laparoscopic approach may be considered the standard of care for benign antero-lateral located lesions, and for left lateral sectorectomy.

HEADINGS - Hepatectomy. Laparoscopy. Liver surgery. Review.

\section{REFERÊNCIAS}

1. Azagra JS, Goergen M, Gilbart E, Jacobs D. Laparoscopic anatomical (hepatic) left lateral segmentectomy-technical aspects. Surg Endosc. 1996; 10: 758-61.

2. Baker TB, Jay CL, Ladner DP, Preczewski LB, Clark L, Holl J, Abecassis MM. Laparoscopy-assisted and open living donor right hepatectomy: a comparative study of outcomes. Surgery. 2009, 146:817-23.

3. Belli G, Fantini C, D'Agostino A, et al. Laparoscopic versus open liver resections for hepatocellular carcinoma in patients with histologically proven cirrhosis: short- and middle- term results. Surg Endosc. 2007; 21: 2004-11.

4. Bryant R, Laurent A, Tayar C, van Nhieu JT, Luciani A, Cherqui D. Liver resection for hepatocellular carcinoma. Surg Oncol Clin N Am. 2008; 17: 607-33.

5. Buell JF, Thomas MT, Rudich S, Marvin M, Nagubandi R, Ravindra KV, Brock G, McMasters KM (2008) Experience with more than 500 minimally invasive hepatic procedures. Ann Surg 248: 475-486

6. Chang S, Laurent A, Tayar C, Karoui M, Cherqui D. Laparoscopy as a routine approach for left lateral sectionectomy. Br J Surg. 2007; 94: 58-63.

7. Cherqui D, Husson E, Hammoud R, Malassagne B, Stéphan F, Bensaid S, Rotman N, Fagniez PL. Laparoscopic liver resections: a feasibility study in 30 patients. Ann Surg. 2000; 232: 753-62.

8. Cherqui D, Laurent A, Mocellin N, Tayar C, Luciani A, Van Nhieu JT, Decaens T, Hurtova M, Memeo R, Mallat A, Duvoux C. Liver resection for transplantable hepatocellular carcinoma: long-term survival and role of secondary liver transplantation. Ann Surg. 2009 Nov;250(5):738-46.

9. Cho JY, Han HS, Yoon YS, Shin SH. Feasibility of laparoscopic liver resection for tumors located in the posterosuperior segments of the liver, with a special reference to overcoming current limitations on tumor location. Surgery 2008 ; 144: $32-8$.

10. Chowbey PK, Shah S, Khullar R, Sharma A, Soni V, Baijal M, Vashistha A, Dhir A. Minimal access surgery for hydatid cyst disease: laparoscopic, thoracoscopic, and retroperitoneoscopic approach. J Laparoendosc Adv Surg Tech A. 2003; 13: 159-65.

11. Dagher I, Lainas P, Carloni A, Caillard C, Champault A, Smadja C, Franco D. Laparoscopic liver resection for hepatocellular carcinoma. Surg Endosc. 2008; 22: 372-8.
12. Descottes B, Glineur D, Lachachi F, Valleix D, Paineau J, Hamy A, Morino M, Bismuth H, Castaing D, Savier E, Honore P, Detry O, Legrand M, Azagra JS, Goergen M, Ceuterick M, Marescaux J, Mutter D, de Hemptinne B, Troisi R, Weerts J, Dallemagne B, Jehaes C, Gelin M, Donckier V, Aerts R, Topal B, Bertrand C, Mansvelt B, Van Krunckelsven L, Herman D, Kint M, Totte E, Schockmel R, Gigot JF. Laparoscopic liver resection of benign liver tumors. Surg Endosc 2003; 17: 23-30.

13. Descottes B, Lachachi F, Sodji M, Valleix D, Durand-Fontanier S, Pech de Laclause B, Grousseau D. Early experience with laparoscopic approach for solid liver tumors: initial 16 cases. Ann Sur. 2000; 232: 641-5.

14. Ferzli G, David A, Kiel T. Laparoscopic resection of a large hepatic tumor. Surg Endosc. 1995; 9: 733-5.

15. Fong Y, Brennan MF, Turnbull A, et al. Gallbladder câncer discovered during lapascopic surgery - potential for iatrogenic dissemination. Arch Surg. 1993; 128:1054-6.

16. Gagner M, Rheault M, Dubuc J. Laparoscopic partial hepatectomy for liver tumor. Surg Endosc. 1992; 6: 99.

17. Gamblin TC, Holloway SE, Heckman JT, Geller DA. Laparoscopic resection of benign hepatic cysts: a new standard. J Am Coll Surg. 2008; 207: 731-6.

18. Gigot JF, Glineur D, Santiago Azagra J, Goergen M, Ceuterick M, Morino M, Etienne J, Marescaux J, Mutter D, van Krunckelsven L, Descottes B, Valleix D, Lachachi F, Bertrand C, Mansvelt B, Hubens G, Saey JP, Schockmel R. Laparoscopic liver resection for malignant liver tumors: preliminary results of a multicenter European study. Ann Surg. 2002; 236: 90-7.

19. Gigot JF, Hubert C, Banice R, Kendrick ML. Laparoscopic management of benign liver diseases: where are we? HPB (Oxford) 2004; 6:197-212.

20. Herman P, Costa ML, Machado MA, Pugliese V, D'Albuquerque LA, Machado MC, Gama-Rodrigues JJ, Saad WA (2005) Management of hepatic hemangiomas: a 14-year experience. J Gastrointest Surg 9: 853-859

21. Herman P, Perini MV, Coelho F, Saad W, D'Albuquerque LA. Half-Pringle maneuver: a useful tool in laparoscopic liver resection. J Laparoendosc Adv Surg Tech A; 2010 Feb;20(1):35-7. 
22. Herman P, Pugliese V, Machado MA, Montagnini AL, Salem MZ, Bacchella T, D'Albuquerque LA, Saad WA, Machado MC, Pinotti HW. Hepatic adenoma and focal nodular hyperplasia: differential diagnosis and treatment. World J Sur. 2000; 24: 372-6.

23. Koffron A, Geller D, Gamblin TC, Abecassis M. Laparoscopic liver surgery: Shifting the management of liver tumors. Hepatology. 2006; 44: 1694-1700.

24. Koffron AJ, Auffenberg G, Kung R, Abecassis M. Evaluation of 300 minimally invasive liver resections at a single institution: less is more. Ann Surg. 2007; 246: 385-92

25. Koffron AJ, Kung R, Baker T, Fryer J, Clark L, Abecassis M. Laparoscopicassisted right lobe donor hepatectomy. Am J Transplant. 2006; 6: 2522-5.

26. Lai EC, Tang CN, Ha JP, Li MK (2009) Laparoscopic liver resection for hepatocellular carcinoma: ten-year experience in a single center. Arch Surg 144(2):143-147

27. Machado MA, Makdissi FF, Galvão FH, Machado MC. Intrahepatic Glissonian approach for laparoscopic right segmental liver resections. Am J Surg.2008;196(4):38-42.

28. Machado MA, Makdissi FF, Surjan RC, Herman P, Teixeira AR, C Machado MC. Laparoscopic resection of left liver segments using the intrahepatic Glissonian approach. Surg Endosc. 2009 Mar 19.

29. Mala T, Edwin B, Gladhaug I, Fosse E, Søreide O, Bergan A, Mathisen O. A comparative study of the short-term outcome following open and laparoscopic liver resection of colorectal metastases. Surg Endosc. 2002; 16:1059-63.

30. Morino M, Morra I, Rosso E, Miglietta C, Garrone C. Laparoscopic vs open hepatic resection: a comparative study. Surg Endosc. 2003; 17: 1914-8.

31. Nguyen KT, Gamblin TC, Geller DA. World Review of Laparoscopic Liver Resection-2,804 Patients. Ann Surg. 2009; 250: 831-41.

32. Nguyen KT, Laurent A, Dagher I, et al. Minimally invasive liver resection for metastatic colorectal câncer. A multi-institutional, international reporto f safety, feasibility, and early outcomes. Ann Surg 2009; 250: 842-8.
33. O'Rourke N, Shaw I, Nathanson L, Martin I, Fielding G. Laparoscopic resection of hepatic colorectal metástases. HPB. 2004; 6: 230-5.

34. Polignano FM, Quyn AJ, de Figueiredo RS, Henderson NA, Kulli C, Tait IS Laparoscopic versus open liver segmentectomy: prospective, case-matched, intention-to-treat analysis of clinical outcomes and cost effectiveness. Surg Endosc. 2008; 22: 2564-70.

35. Saint Marc O, Cogliandolo A, Piquard A, Famà F, Pidoto RR. Early experience with laparoscopic major liver resections: a case-comparison study. Surg Laparosc Endosc Percutan Tech. 2008; 18: 551-5.

36. Santambrogio R, Opocher E, Ceretti AP, et al. Impact of intraoperative ultrassonography in laparoscopic liver surgery. Surg Endosc. 2007; 21(2): 181-8

37. Soubrane O, Cherqui D, Scatton O, Stenard F, Bernard D, Branchereau S, Martelli H, Gauthier F. Laparoscopic left lateral sectionectomy in living donors: safety and reproducibility of the technique in a single center. Ann Surg. 2006; 244: 815-20.

38. Troisi R, Montalti R, Smeets P, Van Huysse J, Van Vlierberghe H, Colle I, De Gendt S, de Hemptinne B. The value of laparoscopic liver surgery for solid benign hepatic tumors. Surg Endosc. 2008; 22: 38-44.

39. Vigano L, Laurent A, Tayar C, Tomatis M, Ponti A, Cherqui D. The learning curve in laparoscopic liver resection: improved feasibility and reproducibility. Ann Surg. 2009 Nov;250(5):772-82.

40. Vigano L, Tayar C, Laurent A, Cherqui D. Laparoscopic liver resection: a systematic review. J Hepatobiliary Pancret Surg. 2009; 16: 410-21.

41. Watson DI. Laparoscopic treatment of gastro-oesophageal reflux disease. Best Pract Res Clin Gastroenterol. 2004; 18: 19-35.

Fonte de financiamento: não há Conflito de interesse: não há Recebido para publicação: 14/05/2009 Aceito para publicação: 01/10/2009 